\title{
Quelle sorte de majorité, ou comment déterminer les lauréats d'un concours?
}

\author{
Louis Laurencelle ${ }^{\mathrm{a}, \bigotimes}$ \\ ${ }^{\mathrm{a}}$ Université du Québec à Trois-Rivières, Canada
}

\begin{abstract}
Determining the final ranking of a set of candidates individually classified by two or more judges is carried out using different proposed rules, each rule giving a somewhat original and sometimes paradoxical result. The known calculation rules are defined, exemplified and analyzed, as well as some new ones. Finally, pragmatic suggestions to help obtain a satisfying outcome are given.
\end{abstract} Acting Editor $\square$ Denis Cousineau (Uni-

Keywords @ Majority calculation, Condorcet’s paradox, rank pooling rules.

\section{louis.laurencelle@gmail.com}

\author{
$L L:$ na
}

10.20982/tqmp.14.4.p235

\section{Introduction}

Il existe différentes sortes de concours dans lesquels des candidats s'affrontent, comme il existe différentes modalités pour comparer et évaluer les concurrents. L'évaluation peut procéder par une mesure, comme dans la compétition sportive d'un sprint pour laquelle le temps, noté en centièmes de seconde, servira à classer les coureurs, ou comme l'examen objectif de connaissances en vue d'un emploi, qui permet d'attribuer un pourcentage de réussite à chaque postulant, éventuellement lui attribuer un rang par rapport aux autres et décider de son admission.

Dans certains cas cependant, l'évaluation reste plus qualitative, voire subjective, même alors qu'elle est appuyée sur des critères d'appréciation convenus, et elle est effectuée par un juge ou par quelques juges : c'est le cas, par exemple, de certaines performances aux Jeux olympiques et lors d'entrevues d'évaluation pour un emploi. Dans de tels cas, chaque juge attribuera, par exemple, une cote de 1 à 9 pour la performance d'un candidat, la "valeur" de ce dernier étant définie par la moyenne arithmétique des cotes qu'il aura reçues des différents juges : le gagnant (pour la médaille d’or !) sera celui obtenant la moyenne la plus élevée.

Dans d'autres cas, particulièrement ceux où seuls quelques candidats sont en lice, les juges, qui font aussi fonction d'électeurs, procéderont plutôt à leur mise en rang, par ordre de préférence : au lieu d'attribuer une cote numérique aux candidats, chaque juge les considérera tous ensemble et les disposera en ordre, du premier (au rang 1) jusqu'au dernier. Des systèmes semblables de choix électoral sont pratiqués, en tout ou en partie, dans certaines administrations (Duverger, 1951; Martin, 2006), et les problèmes encourus ont donné lieu à différentes résolutions, voire à des élaborations théoriques (Grofman \& Lijphart, 1986; Laurent, Delfosse, \& Frognier, 2004) et des expérimentations (p. ex., Dolez \& Laurent, 2010). Occasionnellement, les classements des candidats par les juges, ou électeurs, peuvent concorder entre eux, auquel cas le lauréat, au rang 1, est tout désigné, tout comme le sont le 2 e gagnant, le $3 \mathrm{e}$, etc. Toutefois, dans le cas général, les classements vont différer d'un juge à l'autre, pour des raisons qui échappent à l'analyse mathématique et qui se fondent sur le principe du libre arbitre, voire de la liberté démocratique. Dans le cas de classements plus ou moins discordants, lequel ou lesquels des candidats devrait-on sélectionner ou, dans une formulation plus générale, comment devrait-on combiner en un seul ordre de préférences sociales les préférences individuelles exprimées par les juges?

Pour autant que l'on reconnaisse à chaque juge, ou chaque électeur, une importance égale, comme dans un processus électoral démocratique, c'est le principe de majorité qui est universellement admis à titre axiomatique : le candidat, ou l'ordre des candidats, qui reçoit la faveur 
d'une majorité de votants doit être préféré. Cependant, en considérant les classements multiples et discordants qui apparaissent d'un électeur à l'autre, il est nécessaire de traduire en une règle de compilation spécifique ledit principe de majorité. Comme on le verra, on peut former plusieurs règles différentes, qui donnent parfois aussi des résultats différents, selon différentes interprétations du principe de majorité. Quelle sorte de majorité voulonsnous?

\section{Le principe de Condorcet et la règle de majorité binaire}

Prenons l'exemple suivant, avec 3 juges et 4 candidats dénotés $a, b, c$ et $d$ ( $x>y$ indique que " $x$ ” est préféré à “ $y$ ") : Exemple 1. Préférences de 3 juges pour 4 candidats

$\begin{array}{ll}\text { Juge 1: } & a>b>c>d \\ \text { Juge 2: } & b>a>c>d \\ \text { Juge 3: } & a>c>b>d\end{array}$

La liste des préférences montre à l'évidence que " $d$ " est le dernier candidat (de rang 4), puisque tous les juges s'accordent à ce propos. Qu'en est-il des autres candidats ?

Pour voir clair dans cette liste et afin de déterminer un ordre de préférences sociales, Condorcet (1785; voir aussi Jones, Radcliff, Taber, and Timpone, 1995 et Wikipédia, 2018a) propose la règle de la majorité (de préférence) binaire:

Règle de Condorcet : Est déclaré gagnant celui qui, comparé à chacun des autres candidats, aura été préféré un nombre majoritaire de fois.

Cette règle, qui semble être admise comme fondamentale sans toutefois être appliquée généralement dans les systèmes électoraux publics (Felsenthal \& Machover, 1992), demande qu'on compile les préférences individuelles par paires de candidats, tel que représenté au Tableau 1.

Pour 3 juges, la majorité requiert 2 voix, d'où il appert que le candidat "a" "bat" les 3 autres, puisqu'il est majoritaire par rapport aux trois autres candidats, et il sera déclaré gagnant. La règle peut aussi s’appliquer hiérarchiquement: après l'élection du gagnant "a” (au rang 1), le tableau montre que "b" l'emporte sur "c" et "d" pour le rang 2 et qu'enfin “c” l'emporte sur “d”, d'où un ordre de préférences sociales à la Condorcet de $\mathrm{a}>\mathrm{b}>\mathrm{c}>\mathrm{d}$.

La règle de la majorité binaire, qui peut sembler naturelle pour les exemples simples, se complique facilement et engendre rapidement des difficultés, l'une d'elles étant désignée "paradoxe de Condorcet” (Wikipédia, 2018b). En voici un petit exemple, avec 3 juges et 3 candidats.
Exemple 2. Préférences de 3 juges pour 3 candidats illustrant le paradoxe de Condorcet

$\begin{array}{ll}\text { Juge 1: } & a>b>c \\ \text { Juge 2: } & b>c>a \\ \text { Juge 3: } & c>a>b\end{array}$

Le tableau des préférences binaires montrera ici que , au total, $\mathrm{a}>\mathrm{b}, \mathrm{b}>\mathrm{c}$ et $\mathrm{c}>\mathrm{a}$ : nous avons donc un ordre non transitif, soit $\mathrm{a}>\mathrm{b}>\mathrm{c}>\mathrm{a}$, avec, de plus, l'équivalence des candidats à travers les préférences individuelles des juges: l'élection ici serait impossible.

En admettant que les préférences individuelles sont transitives, ${ }^{1}$ l'agrégation de celles-ci en un ordre global risque d'engendrer plus d'une intransitivité, voire plus d'une anomalie, dès que les préférences individuelles sont non concordantes. Le paradoxe de Condorcet en est un exemple, et il est une simple répercussion mathématique du caractère statistique, on pourrait dire stochastique, du processus électoral.

\section{Le théorème d'impossibilité d'Arrow et les règles pal- liatives}

Considérant un ensemble de préférences individuelles non concordantes, Arrow (Arrow, 1951; Wikipédia, 2018c) a démontré dans un théorème l'impossibilité d'une règle d'agrégation qui produise un ordre de préférences sociales indiscutable. Non seulement la simple règle de Condorcet est susceptible de produire un résultat paradoxal mais toute règle connue ou à inventer y est aussi sujette.

Cette impossibilité d'une règle universelle relativise sérieusement le principe de la majorité binaire de Condorcet, voire de toute majorité, et elle ouvre en même temps la porte à une prolifération de règles ad hoc, lesquelles n’ont pas manqué d'apparaître. Certaines sont des prolongements de la règle de Condorcet tandis que d'autres s'en écartent tout à fait. Nous en examinerons quelques-unes : voir notamment Felsenthal and Machover (1992) et Felsenthal (2010) pour une présentation détaillée des règles de type Condorcet. Les deux exemples suivants serviront de repères.

\section{Exemple 3. Préférences de 3 juges pour 6 candidats (ici,} la majorité est de 2)

$\begin{array}{ll}\text { Juge 1: } & e>a>c>d>f>b \\ \text { Juge 2: } & c>f>b>e>d>a \\ \text { Juge 3: } & a>b>d>f>e>c\end{array}$

\footnotetext{
${ }^{1}$ Une admission qui n’est pas sans poser problème, puisque les juges, ou les électeurs, peuvent mettre en œuvre différents critères afin de classer les candidats et, notamment, peuvent préférer le candidat “x” à au candidat "y” pour un certain critère, et préférer "y” à “z” pour un autre critère. À toutes fins pratiques, nous admettrons tout de même la transitivité de surface de l'ordre des préférences individuelles, abandonnant la question de la pluralité des critères aux politologues et aux psychologues sociaux.
} 
Table 1 - Préférences binaires des exemples 1, 3, et 4 montrant le nombre de juges qui préfèrent le candidat indiqué en rangée par rapport à celui indiqué en colonne

\begin{tabular}{|c|c|c|c|c|}
\hline \multicolumn{5}{|c|}{ Exemple 1} \\
\hline & $\mathrm{a}$ & $\mathrm{b}$ & $\mathrm{C}$ & $\mathrm{d}$ \\
\hline$a>$ & - & 2 & 3 & 3 \\
\hline$b>$ & 1 & - & 2 & 3 \\
\hline$c>$ & 0 & 1 & - & 3 \\
\hline$d>$ & 0 & 0 & 0 & - \\
\hline
\end{tabular}

\begin{tabular}{ccccccc}
\multicolumn{7}{c}{ Exemple 3 } \\
\hline & a & b & c & d & e & f \\
a $>$ & - & 2 & 2 & 2 & 1 & 2 \\
$\mathrm{~b}>$ & 1 & - & 1 & 2 & 2 & 1 \\
$\mathrm{c}>$ & 1 & 2 & - & 2 & 1 & 2 \\
$\mathrm{~d}>$ & 1 & 1 & 1 & - & 1 & 2 \\
$\mathrm{e}>$ & 2 & 1 & 2 & 2 & - & 1 \\
$\mathrm{f}>$ & 1 & 2 & 1 & 1 & 2 & - \\
\hline
\end{tabular}

\begin{tabular}{|c|c|c|c|c|}
\hline \multicolumn{5}{|c|}{ Exemple 4} \\
\hline & $\mathrm{a}$ & $\mathrm{b}$ & $\mathrm{c}$ & $\mathrm{d}$ \\
\hline$a>$ & - & 58 & 72 & 36 \\
\hline $\mathrm{b}>$ & 55 & - & 72 & 57 \\
\hline$c>$ & 41 & 41 & - & 57 \\
\hline$d>$ & 77 & 56 & 56 & - \\
\hline
\end{tabular}

Exemple 4. Préférences de 113 juges pour 4 candidats (ici, la majorité est de 57) (exemple emprunté à Felsenthal \& Machover, 1992)

\begin{tabular}{ll}
\hline pour 19 juges: & $\mathrm{a}>\mathrm{b}>\mathrm{c}>\mathrm{d}$ \\
pour 17 juges: & $\mathrm{b}>\mathrm{a}>\mathrm{c}>\mathrm{d}$ \\
pour 21 juges : & $\mathrm{c}>\mathrm{b}>\mathrm{d}>\mathrm{a}$ \\
pour 19 juges : & $\mathrm{d}>\mathrm{a}>\mathrm{b}>\mathrm{c}$ \\
pour 17 juges: & $\mathrm{d}>\mathrm{b}>\mathrm{a}<\mathrm{c}$ \\
pour 20 juges: & $\mathrm{d}>\mathrm{c}>\mathrm{a}>\mathrm{b}$ \\
\hline
\end{tabular}

Règle de Condorcet : Le (premier) gagnant est celui qui reçoit une majorité de voix contre chacun de ses compétiteurs.

Dans l'exemple 3, le candidat "a” gagne contre tous ses compétiteurs sauf “e” ; il n’y a donc pas de gagnant selon cette règle. La règle n'est pas atteinte non plus dans le cas de l'exemple 4.

Règle de Copeland : À l'échec de la règle de Condorcet, le (premier) gagnant est celui qui obtient le plus grand nombre de comparaisons majoritaires.

Le score de Copeland est le nombre de majorités binaires atteintes par chaque candidat. Dans l'exemple 3, ces scores sont de 4, 2, 3, 1, 3, 2, favorisant ainsi le candidat "a" et produisant du même coup l'ordre agrégé $a>(c, e)>(b, f)$ $>d$, les candidats "b" et "f" (score $=2)$ et "c" et "e" (score = 3) ne pouvant être discriminés Pour l'exemple 4, les scores de Copeland sont respectivement 2, 2, 1 et 1, donnant l'ordre agrégé partiel $(\mathrm{a}, \mathrm{b})>(\mathrm{c}, \mathrm{d})$.

Régle de Copeland-Felsenthal-Machover : Pour chaque candidat d'un groupe ayant obtenu un score de Copeland égal, on détermine le plus petit écart de majorité obtenu par candidat, le candidat gagnant étant celui qui enregistre le plus grand de ces écarts.

En cas d'indétermination par la règle de Copeland, Felsenthal et Machover (1992) proposent d'adjoindre à celle-ci une règle subordonnée dite de "la plus petite majorité”. Le principe de cet avenant est de retenir le candidat pour qui la présence dans le groupe Copeland est la moins fragile, la position majoritaire la plus assurée. Dans l'exemple 4, pour le premier groupe Copeland, c.-à-d. (a, b), les candidats "a" et "b" (score = 2) présentent les écarts majoritaires minimaux de $1(=58-57)^{2}$ pour le candidat "a" et $0(=57-57)$ pour le candidat "b", de sorte que le candidat "a” esp préféré. Quant au second groupe, les candidats "c" et "d" (score = 1) montrent les écarts $0(57-57)$ et 20 (77 - 57), de sorte que “d” l'emporte sur “c”. Le tout forme l'ordre social a > b > d > c. La manœuvre proposée n'est pas applicable à l'exemple 3, lequel ne comporte que 3 juges et où les écarts de majorité sont tous égaux.

Règle de Copeland-Dummett : Pour chaque candidat du groupe ayant obtenu un score de Copeland égal, on compte le nombre total de préférences exprimées, et le candidat pour lequel ce nombre est le plus grand est déclaré premier.

Comme pour la règle Copeland-Felsenthal-Machover cidessus, l'avenant apporté par Dummett (1984) a pour but de décider entre des ex aequo de Copeland, en se basant sur le total des préférences exprimées pour chaque candidat (dit "total de Borda") : ces préférences sont compilées comme ci-dessus, et le total requis est la somme de la rangée correspondante du tableau des préférences. Dans l'exemple 4, les candidats $(\mathrm{a}, \mathrm{b}) \mathrm{du}$ premier groupe Copeland ont reçu 166 et 184 voix, de sorte qu'ici "b" l'emporte sur "a” ; pour le second groupe, les candidats comptent 139 et 189 voix, de sorte que "d" l'emporte sur “c”, le tout formant l'ordre social b > a > d > c. Encore une fois, la petitesse de l'exemple 3 inhibe l'application de cette règle. En effet, les ingrédients du groupe (c, e) ont tous deux 3 majorités, et ceux du groupe $(b, f)$ en ont 2 , d'où la sériation complète des candidats ne peut être achevée.

Règle du total comparatif de Borda : L'ordre des candidats est déterminé par le total des préférences binaires compté pour chaque candidat.

Le total de Borda (voir Wikipedia, 2018) est une mesure naturelle de la préférence que les électeurs ont manifestée pour chaque candidat, l'un par rapport à l'autre. Jean-Charles Borda (XVIIIe siècle) lui-même préconisait un système de pointage relatif au rang d'un candidat dans

\footnotetext{
${ }^{2}$ En rappelant que 57 préférences binaires constitue ici la majorité parmi $k=113$ juges.
} 
l'ordre des préférences individuelles de l'électeur. Le système adopté ici, et dans la règle Copeland-Dummett citée plus haut, correspond ni plus ni moins à donner au candidat un pointage égal à l'inverse de son rang, soit $N$ rang: par exemple, pour l'ordre individuel $\mathrm{a}>\mathrm{b}>\mathrm{c}>\mathrm{d}$, les candidats recevraient les points 3, 2, 1 et 0 . Dans l'exemple 3 , les totaux respectifs sont $9,7,8,6,8,7$, produisant encore l'ordre a $>$ (c, e) $>(\mathrm{b}, \mathrm{f})>\mathrm{d}$. Pour l'exemple 4 , les totaux respectifs 166, 184, 139 et 189 produisent l'ordre $d>b>a>$ c.

Règle de la préséance majoritaire : Le premier lauréat est celui qui a été mis en première place par une majorité simple des électeurs, après quoi, une fois écarté, les lauréats suivants sont trouvés selon la même procédure.

Règle naturelle, c'est celle qui est implicitement mise en application pour la désignation d'un seul gagnant, le candidat placé en tête de liste par un électeur étant presque certainement celui qu'il aurait choisi dans une procédure exigeant une seule désignation. Pour notre exemple 3, qui avec 3 juges réclame une majorité élective de 2, aucun candidat n'obtient deux fois le premier rang. Une solution palliative consiste à cumuler progressivement les rangs, de 1 à $N$, afin d'y repérer un candidat majoritaire, les cas ex aequo étant résolus en fonction de leur rang moyen obtenu. Ainsi, en considérant les deux premiers rangs, le candidat "a" ressort "majoritaire" et se voit attribuer la première place. Cela fait et ajoutant le troisième rang, les candidats "b" et "c" se disputent la seconde place, "c" l'emportant grâce à son rang moyen $(=2,0)$ plus bas que celui de $\mathrm{b}(=$ $2,5)$, ainsi de suite. Ce processus de préséance aboutit à l'ordre social a > c > b > e > f $>d$. Quant à l'exemple 4, nous obtenons directement la séquence $\mathrm{d}>\mathrm{b}>\mathrm{a}>\mathrm{c}$.

Règle du rang moyen : Chacun des $N$ candidats étant classé par chaque juge selon un rang, de 1 à $N$, l'ordre des candidats est déterminé par la moyenne arithmétique de leurs rangs reçus.

Procédure naïve, qui vient naturellement à l'idée pour combiner des informations statistiques de plusieurs sources, le rang moyen est sans doute la procédure la plus commune à être appliquée. Le numéro du "rang”, qui est en fait une variable ordinale, sans propriétés métriques au-delà de la transitivité, est interprété comme un nombre, un pointage, dont la valeur numérique reflète la "grandeur" du candidat. Dénotant par 1 le rang du candidat placé en première place, par 2 celui du second, etc., nous obtenons, pour l'exemple 3 , les rangs moyens 3,00 , $3,67,3,33,4,00,3,33$ et 3,67, résultant en l'ordre social a > (c, e) $>(\mathrm{b}, \mathrm{f})>\mathrm{d}$. Pour l'exemple 4 , les rangs moyens sont $2,53,2,37,2,77$ et 2,33, d'où l'ordre $d>b>a>c$. Noter que cette règle est effectivement la même que celle du total comparatif de Borda, en vertu de la relation (total de Borda $)=($ nombre d'électeurs $) \times(N-$ rang moyen $)$.
Nous proposons enfin deux calculs inspirés de principes de classement différents, la règle de la moyenne géométrique des rangs reçus et celle du produit transitionnel des préférences binaires.

Règle du rang géométrique (ou moyenne géométrique des rangs) : Chacun des $N$ candidats étant classé par chaque juge selon un rang, de 1 à $N$, l'ordre des candidats est déterminé par la moyenne géométrique de leurs rangs reçus.

Dans une logique de choix aléatoire, le candidat qui a obtenu le rang 1 parmi $N$ a un mérite probabiliste de $1 / N$ (ce candidat avait 1 chance sur $N$ d'être placé au hasard au rang 1 ou mieux), de même que le candidat de rang $r$ a un mérite probabiliste de $r / N$. Une autre façon de présenter l'argument est celle-ci : tous les $N$ candidats peuvent obtenir le rang $N$ ou mieux, d'où une probabilité de $N / N$, alors que seuls $r$ candidats peuvent obtenir le rang $r(1 \leq r \leq N)$ ou mieux, d'où une probabilité de $r / N$. Les électeurs votant de façon indépendante, la probabilité conjointe des rangs associée à chaque candidat est le produit des probabilités inter-individuelles, $\prod_{j=1}^{k} 1 / r_{j}$ (où $k$ est le nombre de juges, ou électeurs), quantité qui est inversement proportionnelle à la moyenne géométrique des rangs reçus par chaque candidat. Pour l'exemple 3, les rangs "géométriques" des 6 candidats sont 2,29, 3,30, 2,62, 3,91, 2,71 et 3,42, résultant en l'ordre social $\mathrm{a}>\mathrm{c}>\mathrm{e}>\mathrm{b}>\mathrm{f}>$ d. Pour l'exemple 4, les rangs géométriques sont 2,31, 2,18, 2,50, 1,91, d'où l'ordre $\mathrm{d}>\mathrm{b}>\mathrm{a}>\mathrm{c}$.

Règle du produit transitionnel : L'ordre social optimal, avec les rangs des candidats qui le forment, est celui pour lequel le produit des préférences binaires successives est le plus grand.

Nonobstant les rangs individuels des candidats, l'ordre social cherché devrait être, parmi les permutations autorisées de candidats (c.-à-d. celles pour lesquelles chaque transition a un score de préférences non nul), celle dont le produit des scores transitionnels est le plus grand. Noter que ce principe de calcul porte d'abord sur le classement des candidats, leur sériation, et ne s'adresse qu'indirectement à l'élection du ou des meilleurs d'entre eux. Ainsi, dans notre exemple 3, la permutation a b c d e f présente, au tableau des préférences binaires, les scores de transition 2, 1, 2, 1, 1; par exemple, la transition $a \rightarrow b$, notée à l'intersection de la ligne a et la colonne $\mathrm{b}$, apparaît 2 fois, le produit transitionnel, $2 \times 1 \times 2 \times 1 \times 1$ donnant 4 . De la même façon, la permutation a b d f c e présente les scores 2, 2, 2, 1, 1, de produit 8 .. Pour cet exemple, le produit transitionnel le plus grand, 32, correspond à la permutation, et à l'ordre social, a > c > d > f > b > e. Quant à l'exemple 4, le produit maximal, 321552, dénote l'ordre social $d>a>b>c$.

Le tableau 2 donne un aperçu synoptique de 
Table 2 a Synopsis des règles d'élection et des classements résultants dans deux exemples (voir texte)

\begin{tabular}{lcc}
\hline Règle d'élection & Exemple 3 & Exemple 4 \\
\hline Condorcet & - & - \\
Copeland & $\mathrm{a}>(\mathrm{c}, \mathrm{e})>(\mathrm{b}, \mathrm{f})>\mathrm{d}$ & $(\mathrm{a}, \mathrm{b})>(\mathrm{c}, \mathrm{d})$ \\
Copeland-Felsenthal-Machover & $\mathrm{a}>(\mathrm{c}, \mathrm{e})>(\mathrm{b}, \mathrm{f})>\mathrm{d}$ & $\mathrm{a}>\mathrm{b}>\mathrm{d}>\mathrm{c}$ \\
Copeland-Dummett & $\mathrm{a}>(\mathrm{c}, \mathrm{e})>(\mathrm{b}, \mathrm{f})>\mathrm{d}$ & $\mathrm{b}>\mathrm{a}>\mathrm{d}>\mathrm{c}$ \\
Total de Borda & $\mathrm{a}>(\mathrm{c}, \mathrm{e})>(\mathrm{b}, \mathrm{f})>\mathrm{d}$ & $\mathrm{d}>\mathrm{b}>\mathrm{a}>\mathrm{c}$ \\
Préséance & $\mathrm{a}>\mathrm{c}>\mathrm{b}>\mathrm{e}>\mathrm{f}>\mathrm{d}$ & $\mathrm{d}>\mathrm{a}>\mathrm{b}>\mathrm{c}$ \\
Rang moyen & $\mathrm{a}>(\mathrm{c}, \mathrm{e})>(\mathrm{b}, \mathrm{f})>\mathrm{d}$ & $\mathrm{d}>\mathrm{b}>\mathrm{a}>\mathrm{c}$ \\
Rang géométrique & $\mathrm{a}>\mathrm{c}>\mathrm{e}>\mathrm{b}>\mathrm{f}>\mathrm{d}$ & $\mathrm{d}>\mathrm{b}>\mathrm{a}>\mathrm{c}$ \\
Produit transitionnel & $\mathrm{a}>\mathrm{c}>\mathrm{d}>\mathrm{f}>\mathrm{b}>\mathrm{e}$ & $\mathrm{d}>\mathrm{a}>\mathrm{b}>\mathrm{c}$ \\
\hline
\end{tabular}

l'application des diverses règles d'élection appliquées à nos deux exemples.

\section{Discussion et conclusion}

Dans un premier temps, nous considérons certaines propriétés comparatives des différentes règles proposées et discutons ensuite du principe de "majorité électorale" et de sa mise en œuvre dans un contexte statistique réel.

Le but d'une règle électorale, rappelons-le, est de départager les candidats et leur attribuer un rang respectif qui reflète de manière démontrable et crédible les préférences exprimées par les électeurs ou les juges d'un concours. Or, le caractère statistique, c'est-à-dire objectivement stochastique, d'un scrutin rend la chose difficile, particulièrement dans le cas d'un vote multinominal dans lequel chaque votant place les candidats dans son ordre de préférence. Les principes de compilation électorale à respecter seront donc:

1. d'imposer d'emblée et jusqu'au terme du processus le principe de majorité électorale;

2. d'utiliser une règle convenue, explicite et raisonnable pour ordonner les candidats sur la base des votes reçus;

3. en cas d'indécision sur la règle convenue, d'appliquer une seconde règle à titre de règle auxiliaire afin de trancher l'indécision;

4. en cas d'égalité statistique absolue, de déclarer ex aequo les candidats concernés et reprendre le vote au besoin.

Dans la procédure suggérée, et en admettant que chacune des règles ci-dessus a son mérite et ses arguments, il est intéressant d'en comparer l'aptitude à éluder les cas d'indécision.

Dès l'abord, la règle princeps de Condorcet, qui stipule que le candidat gagnant (c.-à-d. au rang 1) devra avoir été préféré à chacun des autres par une majorité d'électeurs, se révélera souvent irréaliste, nous forçant à recourir à une règle subsidiaire. Or, toutes les autres règles énumérées répercutent la règle de Condorcet : si la condition de Condorcet s'applique, quel que soit le rang du can- didat, toutes les règles présentées fourniront le même ordre de préférences sociales que celle de Condorcet. Dans ce contexte, quels sont les mérites relatifs des 8 règles concernées?

La règle de Copeland, qui ordonne les candidats selon le nombre de majorités binaires remportées, apparaît bien fragile, puisque ces majorités de préférences peuvent se jouer et se jouent sur un seul électeur et ne tiennent pas compte du poids des majorités. Prenons l'exemple 4, et la règle Copeland-Felsenthal-Machover, qui produit l'ordre $\mathrm{a}>\mathrm{b}>\mathrm{d}>\mathrm{c}$. Supposons que, parmi les 113 électeurs, l'un modifie son ordre $\mathrm{c}>\mathrm{b}>\mathrm{d}>\mathrm{a}$ pour l'ordre $\mathrm{d}>\mathrm{b}>$ $\mathrm{a}>\mathrm{c}$; la règle produira alors l'ordre global $\mathrm{d}>\mathrm{a}>\mathrm{b}>$ c. Remarquons ici que, de sa troisième position d'après le classement Copeland-Felsenthal-Machover, le candidat “d” devient un lauréat de Condorcet, raflant toutes les majorités! La même critique de fragilité s’applique à la règle Copeland-Dummett.

Le risque de majorité fragile concerne-t-il aussi la règle de Préséance, considérant que la place d'un candidat dépend d'une compilation incomplète des préférences? En fait, pour désigner, par exemple, le candidat de rang 1, il n’y a qu'une seule majorité qui entre en jeu, ici une majorité simple, à savoir le candidat qui a été placé en tête de liste par le plus grand nombre d'électeurs : cette majorité de position est patente, simple et crédible : le plus grand nombre d'électeurs retrouvera son candidat à la position voulue. La règle s'étendant facilement aux rangs ultérieurs, le principe de majorité (simple) et l'apparence du principe de majorité sont mis en valeur.

Nous avons vu que le total de Borda (= somme des préférences binaires reçues par un candidat) et le rang moyen forment le même indice statistique, et nous croyons (sans enquête) qu'il s'agit ici d'une règle très communément exploitée dans les petites organisations. Or, le calcul du rang moyen suppose une métrique, une unité de mesure, selon laquelle les rangs “ $1,2,3, \ldots$ ” ont une valeur quantitative et que, par exemple, le candidat ayant reçu deux fois le rang 2 équivaut précisément à celui ayant reçu 
les rangs 1 et 3. Cette extrapolation métrique n'a pas de fondement et n'est intéressante que pour sa commodité. ${ }^{3}$

Quant aux critères de justification mathématique et de commodité, le rang géométrique semble l'emporter sur le rang moyen. D'abord, la métrique de cette règle est de support probabiliste, l'agrégation des préférences (en appliquant le théorème sur la probabilité d'occurrence conjointe d'événements indépendants) s'en trouvant justifiée. De plus, il s'avère que le risque d'indécision est sensiblement moindre avec la moyenne géométrique. En fait, dans un système aléatoire, le nombre éventuel de valeurs différentes du rang moyen serait de $k \times N-k+1$, où $k$ et $N$ dénotent respectivement le nombre de juges (ou électeurs) et de candidats, alors qu'il serait plutôt d'ordre $O\left(N^{k}\right)$ pour le rang géométrique. ${ }^{4}$ Par exemple, pour 4 candidats et 6 juges, nous comptons 19 rangs moyens et 145 rangs géométriques distincts. Dans notre Exemple 4, les deux règles produisent le même ordre complet, alors que le rang géométrique achève la sériation de l'exemple 3. Quelle est, en général, la probabilité que la règle géométrique gagne sur la règle du rang moyen pour produire un classement complet ? L'étude reste à faire.

Finalement, la règle du produit transitionnel, qui partage les mêmes vertus discriminantes que la règle géométrique, et pour la même raison, se base sur une mesure de "solidité" d'un réseau hiérarchique : la force transitionnelle du réseau est proportionnelle au produit des forces de ses connexions. Par contre, cette règle, qui s'appuie sur la solidité de l'ensemble hiérarchique, ne valorise pas la préséance de rang, ce qui explique, par exemple, qu'un candidat apparemment défavorisé, le candidat "e" dans notre exemple 3, prend du galon en remontant au rang 3 dans la série résultante.

Il n'y a pas de règle absolue, c'est-à-dire de règle qui, lorsqu'elle est appliquée à un ensemble non cohérent de préférences individuelles, produira un ordre de préférences sociales satisfaisant tous les critères raisonnables. À défaut d'une majorité claire des préférences individuelles, ${ }^{5}$ il nous faut envisager l'obtention d'un ordre de préférences sociales comme un problème d'estimation statistique, en imposant le respect des quatre principes énoncés ci-dessus.

Dans le contexte des principes mentionnés, notamment en vertu du caractère explicite et raisonnable de la méthode de compilation, les règles du Total de Borda, de Copeland-Dummett et du Produit transitionnel semblent mal qualifiées. Par ailleurs, malgré sa pauvre légitimité mathématique, la règle du Rang moyen a une valeur d'évidence et peut servir à pallier et racheter, pour ainsi dire, la règle de Copeland-Dummett, puisque le total de Borda et le rang moyen sont en fait une même statistique. À un second niveau, le critère des préférences binaires de Condorcet et les règles de Copeland qui s'y rattachent nous semblent peu convaincants : bien sûr, un ordre transitif de candidats, tel que $\mathrm{a}>\mathrm{b}>\mathrm{c}>\mathrm{d}$, implique et suppose les inégalités intercalaires, telles que $a>c$ ou $b>$ $\mathrm{d}$, inégalités sur lesquelles les préférences binaires sont aussi établies. Mais l'électeur ne construit vraisemblablement pas son ordre de cette façon ; plutôt, à partir de critères objectifs et subjectifs, l'électeur va assigner le rang de chaque candidat, en indiquant notamment le candidat qu'il souhaite voir au rang 1 . Pour cette raison, la règle de Préséance majoritaire nous paraît la plus limpide, en fait la plus démocratique en apparence, avec la règle du Rang géométrique, qui a la même vertu d'évidence que le Rang moyen tout en bénéficiant d'une base mathématique plus sûre.

Quelle que soit la règle utilisée, ou les règles dans le cas où une règle auxiliaire doive être ajoutée en cas d'indécision, il importe surtout que l'entière procédure de compilation soit établie à l'avance et connue des juges et de l'organisme qui gère le concours, ce dans le but d'éviter tout dérapage et toute occasion d'arbitraire durant le processus de jugement, limitant ainsi les risques de contestation de la part des juges autant que des candidats.

\section{References}

Arrow, K. J. (1951). Social choice and individual values. New York: Wiley.

de Condorcet, N. (1785). Essai sur l'application de l'analyse à la probabilité des décisions rendues à la pluralité des voix. Paris: l'Imprimerie Royale.

Dolez, B., \& Laurent, A. (2010). La magnitude, facteur décisif? les élections européennes de 2004 en france et les effets du changement de mode de scrutin. Revue internationale de politique comparée, 17(3), 175-193.

Dummett, M. (1984). Voting procedures. Oxford: Clarendon Press.

Duverger, M. (1951). Les partis politiques. Paris: Armand Colin.

Felsenthal, D. S. (2010). Review of paradoxes afflicting various voting procedures where one out of $m$ candidates $(\mathrm{m}>2)$ must be elected, Château du Baffy, France, 54 . Voting Power im Practice.

\footnotetext{
${ }^{3}$ Une critique semblable s'adresserait au Total de Borda, dans lequel on additionne des préférences touchant des couples différents de candidats. Ainsi, est-ce que 10 préférences $(a>b)$ ont la même valeur que 10 préférences $(a>d)$, alors même que les votants auraient tous fourni un ordre $a>b>$ $c>d$ ? L’agrégation de ces préférences binaires n’a donc elle-même aucune justification métrologique.

${ }^{4}$ Pour le cas de $J=2$ juges, le nombre de rangs géométriques différents est $2 N-1$, égal ici à celui des rangs moyens.

${ }^{5}$ Donnons comme exemple le cas de 100 électeurs et 4 candidats (a, b, c, d), avec 51 électeurs (la majorité) produisant l'ordre a > b > c > d et les 49 autres donnant n'importe quelles autres permutations, cas pour lequel l'ordre de la majorité prévaudra selon toutes les règles.
} 
Felsenthal, D. S., \& Machover, M. (1992). After two centuries, should condorcet's voting procedure be implemented? Behavioral Science, 37, 1-24. doi:10.1002/bs. 3830370403

Grofman, B., \& Lijphart, A. (1986). Electoral laws and their political consequences. New York: Agathon Press.

Jones, B., Radcliff, B., Taber, C., \& Timpone, R. (1995). Condorcet winner and the paradox of voting : probability calculations for weak preference orders. American Political Science Review, 89, 137-144. doi:10.2307/ 2083080

Laurent, A., Delfosse, P., \& Frognier, A. P. (2004). Les systèmes électoraux permanences et innovations. Paris: L'Harmattan.

Martin, P. (2006). Les systèmes électoraux et les modes de scrutin (3e édition). Paris: Clefs Montchrestien.
Wikipedia. (2018). Borda count - Wikipédia, the free encyclopedia. Retrieved, from https://en.wikipedia.org/ wiki/Borda_count

Wikipédia. (2018a). Méthode de Condorcet - Wikipédia, l'encyclopédie libre. Retrieved, from http : / / fr . wikipedia.org/w/index.php?title =M\%C3\%A9thode_ de_Condorcet\&oldid=149116702

Wikipédia. (2018b). Paradoxe de Condorcet — Wikipédia, l'encyclopédie libre. Retrieved, from http : / / fr . wikipedia . org / w / index . php ? title = Paradoxe _ de _ Condorcet\&oldid=147661741

Wikipédia. (2018c). Théorème d'impossibilité d'Arrow Wikipédia, l'encyclopédie libre. Retrieved, from http: //fr.wikipedia.org/w/index.php?title=Th\%C3\%A9or\% C3\%A8me_d\%27impossibilit\%C3\%A9_d\%27Arrow\& oldid $=154105117$

\section{Citation}

Laurencelle, L. (2018). Quelle sorte de majorité, ou comment déterminer les lauréats d'un concours? The Quantitative Methods for Psychology, 14(4), 235-241. doi:10.20982/tqmp.14.4.p235

Copyright (C 2018, Laurencelle. This is an open-access article distributed under the terms of the Creative Commons Attribution License (CC BY). The use, distribution or reproduction in other forums is permitted, provided the original author(s) or licensor are credited and that the original publication in this journal is cited, in accordance with accepted academic practice. No use, distribution or reproduction is permitted which does not comply with these terms.

Received: 14/08/2018 Accepted: 02/11/2018 